\title{
IR thermometry for heat and mass transfer analysis of surface drying of fruit
}

\author{
by K. Gottschalk*, Cs. Mészáros**
}

\begin{abstract}
*Leibniz-Institut für Agrartechnik Potsdam-Bornim e.V. (ATB), Max-Eyth-Allee 100, 14469 Potsdam, Germany, kgottschalk@atb-potsdam,.de

**Szent István University, Department of Physics and Process Control, H-2103 Gödöllő, Páter K.u.1, Hungary, Meszaros.Csaba@gek.szie.hu
\end{abstract}

\begin{abstract}
Preceding storage of fruit and potatoes the surface of the fruits must be dried off from moist to prevent microbial activities and to secure long term storage. Most of interests are the physical properties of the diffusive and convective transfer phenomena of vapor between fruit body (surface) and ambient air during surface moist evaporation when ventilating fruit or potato bulks in boxes in a storeroom. The air condition at certain air flow velocity, temperature and humidity, and also the fruit state (surface condition, form, maturity, etc) are the influencing parameters on the evaporation process. The primary effects on the moisture loss in a fruit or potato are the diffusion processes in the ambient air and in the produce material. The environmental conditions can easily be obtained under test. An analytical model gives a tool to investigate the mass loss for a transpiring produce. The model described here is the basis for determining heat and mass transfer from the surface of a spherical fruit to the ambient air. It gives a simple method to determine the environmental influences on the moisture loss. The temperature distribution along a fruit's surface is measured using thermal imaging.
\end{abstract}

\section{Introduction}

During ventilation of the fruit directly with air, the influences of the developed air flow on the evaporation process around single fruit bodies or clusters of fruits is investigated by using an infrared thermographic camera. The effect of surface drying is determined by using the evaporation cooling effect along the surface. The air flow is visualized by image analysis of the thermographic images to discriminate local flow and transfer effects. Respecting the boundary conditions, the distribution of the air humidity, from the surface of the produce to the ambient flowing air can be calculated when identifying the mass loss with mass density flow.

Cooling, drying and storing of fruit or potatoes is accompanied with mass loss, i.e. loss of water which means loss of weight, loss of quality, pecuniary loss, and loss of freshness. Most of interests are the physical properties of the transport and convective transfer phenomena of vapour between fruit body and ambient air, when ventilating fruits in a storeroom. The air with certain velocity, temperature and humidity, and also the fruit state (condition, type, variety, maturity, etc) are the influencing parameters on the transpiration process.

Re-warming of fruits and vegetables after cooling is characterized by heat and mass transfer processes which leads commonly to condensation of water on the produce surface at temperatures below the dew point. This effect may affect the produce quality due to microbial growth at unfavorable environmental conditions. The amount of condensed water is a function of the produce surface temperature and of the surrounding conditions as air temperature, air humidity, and air flow. Under practical conditions both the warming and the condensation is strongly affected by the packaging system used. Depending on the flow conditions close to the produce surface some important basic interactions at single produce items under laboratory conditions were measured. A mathematical model is developed for the determination of the amount of condensed water on fruit surfaces, its re-evaporation and its total dwell time dependent on the environment air conditions. The model describes the heat and mass transfer processes on single fruits. The process of diffusion of humidity in air and proceed of surface temperature is the basis for the model.

\section{Air flow modelling and measuring}

The air flow pattern is determined while the air condition is varying, i.e. changing of air velocity, air temperature and air humidity. To model the air flow around an obstacle, the method of Computational Fluid Dynamics (CFD) is used. The model is solving the Navier-Stokes equation system in conjunction of the energy equation to determine the associated temperature field. The equation system is discretised by using the Finite Difference Method (FDM). For simplification, the shape of the fruit is approximated to a sphere. 


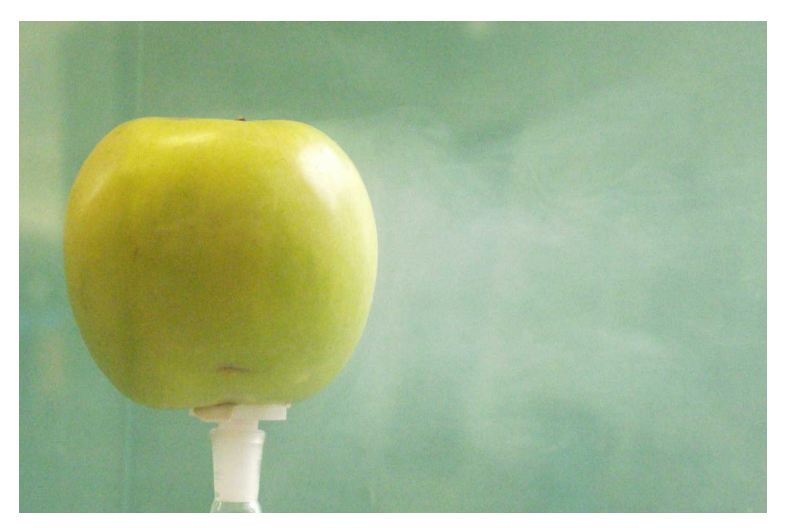

Fig. 1. Visualized air flow around an apple with von Kármán vortex street

The laminar flow around a spherical fruit (figure 1) and within the boundary layer near the surface can be examined with a sufficient accuracy.

Based on the CFD model for the air flow, the air velocity around the object is calculated and compared, figure 2 and figure 3. The boundary layer region is examined for two different Reynolds numbers, for example. Even if the fluctuations are not modelled correctly at the moment, the results come very close to the measured values.

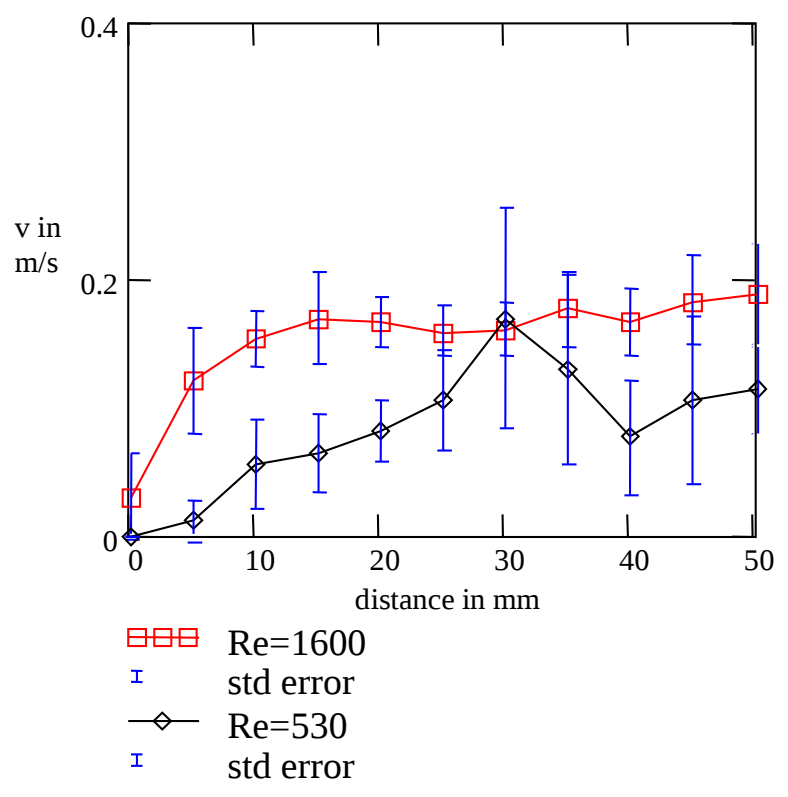

Fig. 2. Measured air flow velocity within the boundary layer of an apple crossways the inflow direction. 


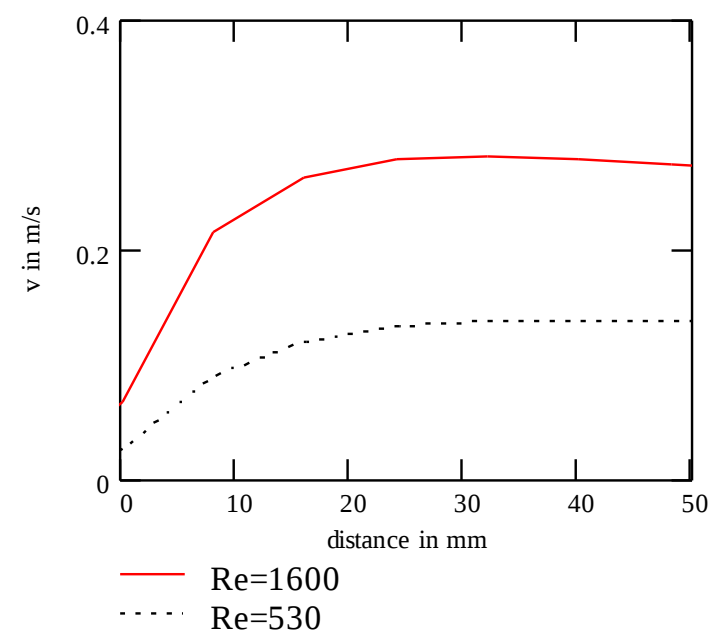

Fig. 3. CFD-modelled air flow velocity within the boundary layer of an apple crossways the inflow direction

\section{Mass transfer}

Investigations were made by a number of authors to determine transpiration coefficients when ventilating a bulk of fruits with conditioned air of fixed properties (e.g. air rate, temperature, humidity). Although, it is still of interest how the air flow is developing around a fruit body when ventilating directly the body and how the transpiration and heat exchange process is influenced by the air flow pattern.

The primary effects on the moisture loss are the diffusion processes in the ambient air and in the produce material. The environmental conditions can easily be obtained under test. An analytical model gives a tool to investigate the mass loss for the transpiration process of the produce. The model described here is an alternative approach to the studies of the transpiration processes that were worked out before, e.g. by Linke [1]. The modelling method described there is a method to determine the environment influences on the moisture loss.

The moisture loss is determined by Linke $[1,2]$

$$
E=\frac{m_{0}-m_{1}}{A \cdot\left(t_{1}-t_{0}\right)}
$$

where $m_{0}$ is the total mass of the fruit with surface area $A$ at start of experiment a time $t_{0}, m_{1}$ is the total mass of the fruit after experimental time $t_{1}$, [2]. The specific mass loss flow $E$ is identified with the mass flow density $j$ into the ambient air at the surface. This phenomena follows the diffusion equation)

$$
j=-D \operatorname{grad} x
$$

with $\mathrm{D}$ as diffusion coefficient. $\mathrm{D}_{\text {air }}=25.6 \cdot 10^{-6} \mathrm{~m}^{2} \cdot \mathrm{s}^{-1}$ for vapour diffusion in dry air, and humidity (water content) $\mathrm{x}$ defined as $\mathrm{x}=\mathrm{m}_{\text {humid air }} / \mathrm{m}_{\text {dry air }}$.

Respecting the boundary conditions for the mass flow density $j$ at the surface of the fruit and for $x$ at a distance $r_{A}$ from the surface with $r_{A} \gg R_{0}$ gives the solution of the diffusion equation for a sphere of radius $R_{0}$ (i.e. in spherical coordinates)

$$
\partial x / \partial t=D_{\text {air }}\left(2 / r \partial x / \partial r+\partial^{2} x / \partial r^{2}\right)=0
$$

for stationary states to

$$
x(r)=x_{R 0}+\left(x_{A}+x_{R 0}\right) \quad\left(1-R_{0} / r\right)
$$

In this model the fruit is idealized to an object with spherical shape.

With

$$
\rho=(1+x) /\left(R_{A}+x R_{V}\right) \cdot p / T
$$


the mass (vapour) flow density in dry air for stationary state can be calculated using

$$
j=-D d \rho / d r=-D d \rho / d x \cdot d x / d r
$$

The solution represents the mass flow from the surface of the fruit into the ambient air at any time, figure 4.

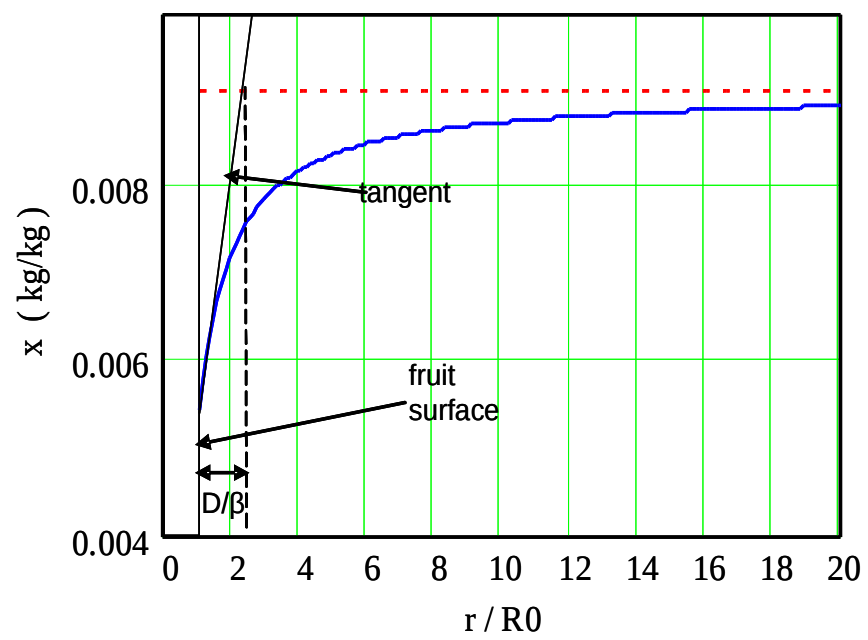

Fig. 4. Example of absolute humidity $x$ in air at distance of $R_{0}$ units from an apple surface

The course of the mass flow density gives an approximation of the boundary layer thickness $\delta$ at air flow velocity equal to zero, i.e. pure diffusion to take place. Hence, the convection term is zero but arises when forced air flow develops. At air flow velocity $w>0$, the boundary layer thickness $\delta$ decreases, which causes the mass transfer coefficient $\beta$ to increase. This process is represented by the Sherwood number

$$
\mathrm{Sh}=\beta \cdot \delta / \mathrm{D}
$$

from which the mass transfer coefficient $\beta$ can be determined, if $\delta$ is known or can be approximated using the boundary layer thickness.

The air humidity $\left(x_{A}\right)$ can easily be measured at a far distance $r_{A}$, while $x_{R 0}$ close to the surface can be assumed to be at the saturation point for the vapour-air mixture within the boundary layer, as long as the surface is wet i.e. covered with a thin water film layer. Using the definition for $\beta$ as

$$
\beta=-D_{\text {air }} \cdot(\partial x / \partial r)_{\text {surface }} /\left(x_{s 0}-x_{\delta}\right)
$$

the ratio $D_{\text {air }} / \beta=\delta / S_{\delta}$ can be determined using the derivative of equ. (4) or taken from the graph (figure 4). ( $x_{s 0}$ air humidity at saturation point on the surface, $x_{\square}$ at saturation point at the boundary layer distance). For the example stated here $D_{\text {air }} / \beta$ is approx. (1.20...1.22) $\times R_{0}$ when neglecting free convection; ( $R_{0}=$ fruit radius).

With this method, $\beta$ was found as approx. $1.8 \cdot 10^{-3} \mathrm{~m} / \mathrm{s}$, while measured in the range of $2.5 \ldots 3.3 \cdot 10^{-3} \mathrm{~m} / \mathrm{s}$. For laminar air flow around a sphere, the Sherwood number is found [3] to be

$$
\mathrm{Sh}=0.644 \mathrm{Re}^{1 / 2} \mathrm{Sc}^{1 / 3}
$$

using the Schmidt number Sc $=v / D$ and $v$ the viscosity, $D$ the diffusion coefficient. By knowing the velocity field around the object, the mass transfer coefficient can be determined.

Using the air-flow velocity distribution around the fruit (sphere) measured (figure 5) or modelled, respectively, we can obtain the (local) mass transfer coefficient $\beta(s)$ for the path s half around the fruit $0 \leq s \leq \pi$, figure 6 . The average

$$
\beta_{\text {total }}=\frac{1}{\pi} \int_{s=0}^{\pi} \beta(s) d s
$$


corresponds to the integral (total) transfer coefficient $\beta$ for the fruit, see above.

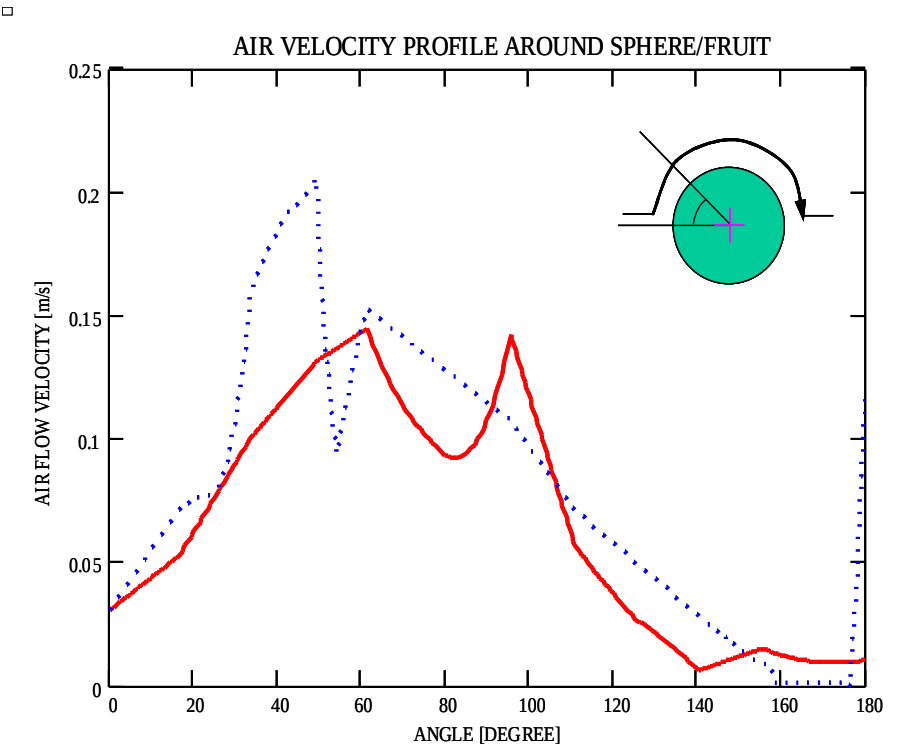

Fig. 5. Measured air flow velocity along a fruit surface; solid line: upper half, dashed line: lower half of fruit

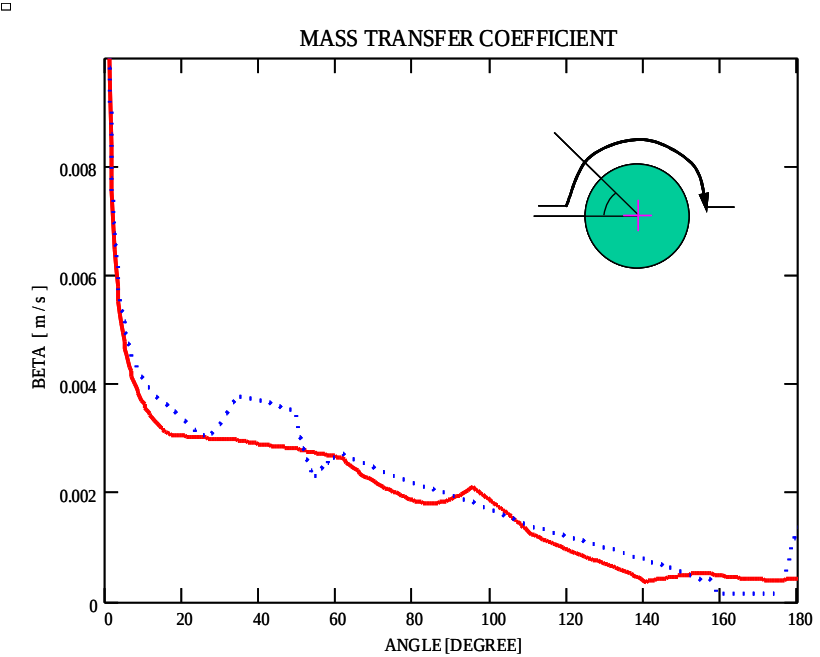

Fig. 6. Mass transfer coefficient $\beta(s)$ along fruit surface; solid: upper half, dashed: lower half of fruit

\section{Heat transfer}

The methodology developed here for the mass transfer is analogous to the heat transfer, if the Nusselt number Nu is taken, similar to the Sherwood number, i.e. 


$$
\mathrm{Nu}=0.664 \operatorname{Re}^{1 / 2} \operatorname{Pr}^{1 / 3}
$$

for an air flow around a sphere with the Prandtl number $\operatorname{Pr}=v / \mathrm{a}$, with $v$ viscosity, a thermal conductivity. For gases, Pr is approx. 0.7 .

By using

$$
\mathrm{Nu}=\alpha \cdot \delta_{\mathrm{T}} / \lambda
$$

with $\alpha$ the thermal transfer coefficient, $\lambda$ the heat conductivity coefficient, and assuming $\delta_{\mathrm{T}} \approx \delta$.

\section{Infrared thermometry}

Thermal imaging (thermometry), using an electronic infrared camera, is a method to determine the drying process when drying off the moisture from a fruit surface $[4,5]$. As an example the temperature distribution along the surface of a wet peach is shown in figure 7. The evaporation of surface moisture lowers the temperature down to wet bulb temperature. As soon the surface is (partially) dried off the temperature increases again to ambient air value which can be seen by the temperature profile, measured with IR camera, figure 8 . The movement of a 'drying zone' from the side of air-flow direction to the opposite side can be well recognized. The temperature distribution development on the surface is dependent on air flow direction and velocity; the drying time is also dependent on air humidity.

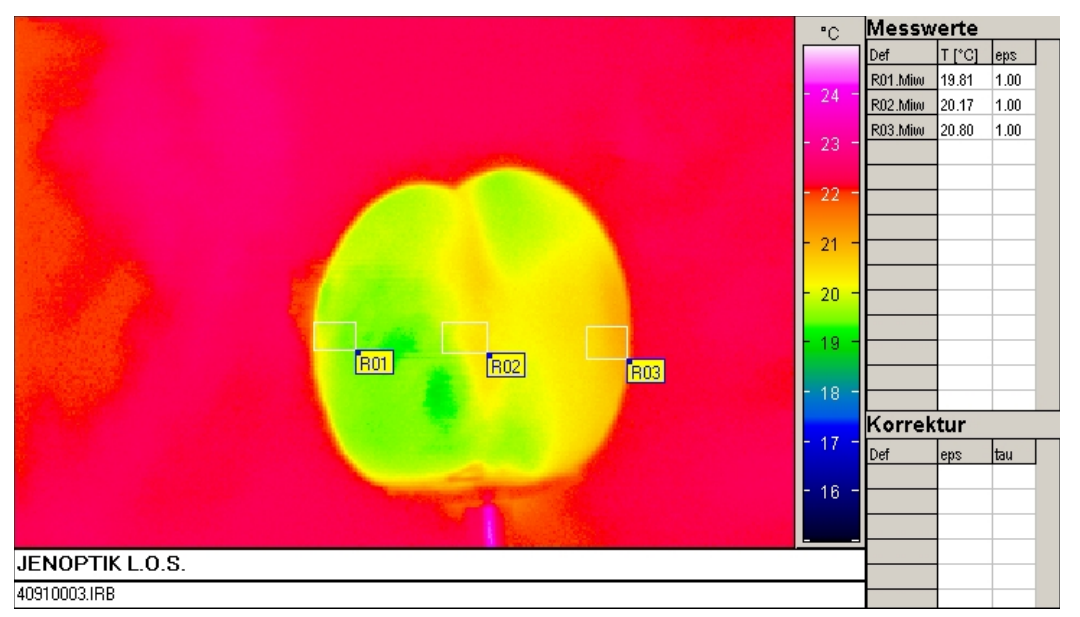

Fig. 7. Temperature distribution on the surface of a peach during drying off surface moisture using infrared thermography; with 3 test points 'R01', 'R02', 'R03'

For example (figure 8), if ambient (air flow) temperature is $25^{\circ} \mathrm{C}$ at $32 \% \mathrm{rH}$, the wet bulb temperature is $14.8^{\circ} \mathrm{C}$. We can see in figure 8 that the wet-bulb temperature is not reached. The reason is that the evaporative cooling is not adiabatic in reality. Besides from the flowing air, heat is also transported from inside the fruit to the surface and takes part of energy delivery (enthalpy) to the evaporation process. To analyse this phenomenon the classical Fourier's heat transfer equation is used. 


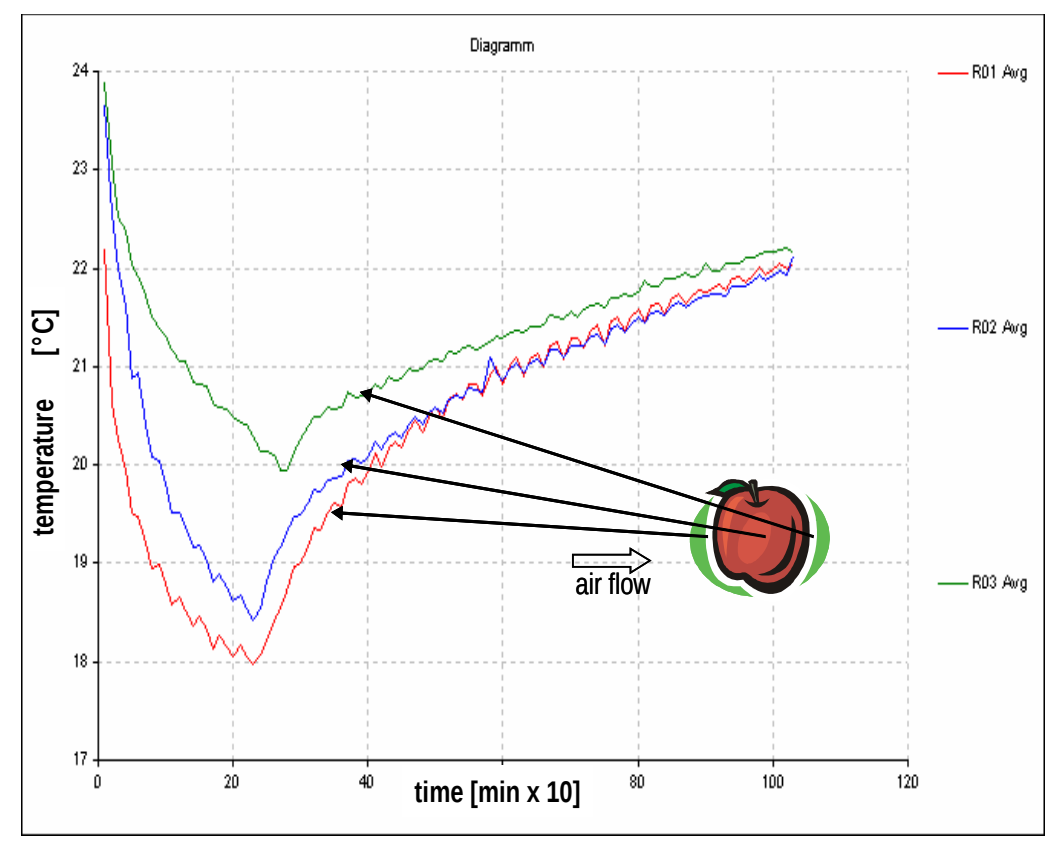

Fig. 8. Infrared measured temperature profile at three local points on the surface of a peach during drying off surface moisture

\section{Evaporation model}

Evaporating water causes cooling the surface down to wet-bulb temperature which is called adiabatic evaporative cooling. Since it should be assumed that heat is not transported from inside the body (fruit, potato tuber etc.) but only from the air flowing over the surface, the process is adiabatic. The wet bulb temperature $\theta_{\mathrm{s}}$ can be calculated using

$$
\theta_{\mathrm{amb}}-\theta_{\mathrm{s}}=\frac{\Delta \mathrm{h}_{\mathrm{v}}\left(\theta_{\mathrm{s}}\right) \cdot\left(\mathrm{Y}_{\mathrm{s}}\left(\theta_{\mathrm{s}}\right)-\mathrm{Y}_{\mathrm{s}}\left(\theta_{\mathrm{amb}}\right) \cdot \mathrm{rH}\right)}{\mathrm{c}_{\mathrm{pg}}+\mathrm{Y} \cdot \mathrm{c}_{\mathrm{pv}}}
$$

The temperature $T_{s 0}$ is the value on the moist surface of the fruit at saturation point, i.e. water is condensed and the air closest to the surface is saturated with humidity $(\mathrm{rH}=100 \%)$, at the temperature $\mathrm{T}_{\mathrm{s} 0}$. This temperature is measured with infrared camera on a peach during ventilation with air at $25{ }^{\circ} \mathrm{C}, 33 \% \mathrm{rH}$ and velocity $\mathrm{w}$ of $0.1 \mathrm{~m} \cdot \mathrm{s}^{-1}$, figure 8.

The temperature distribution development on the surface is dependent on air flow direction and velocity, as well as on temperature and air humidity. The drying time, i.e. the time until the surface moist is totally removed, can be well recognized as the point when all local temperatures on the fruit surface have converged to the ambient or fruit temperature. Using the model described here in the 'heat transfer' section we can see sufficient agreement with the measurements (figure 9). 


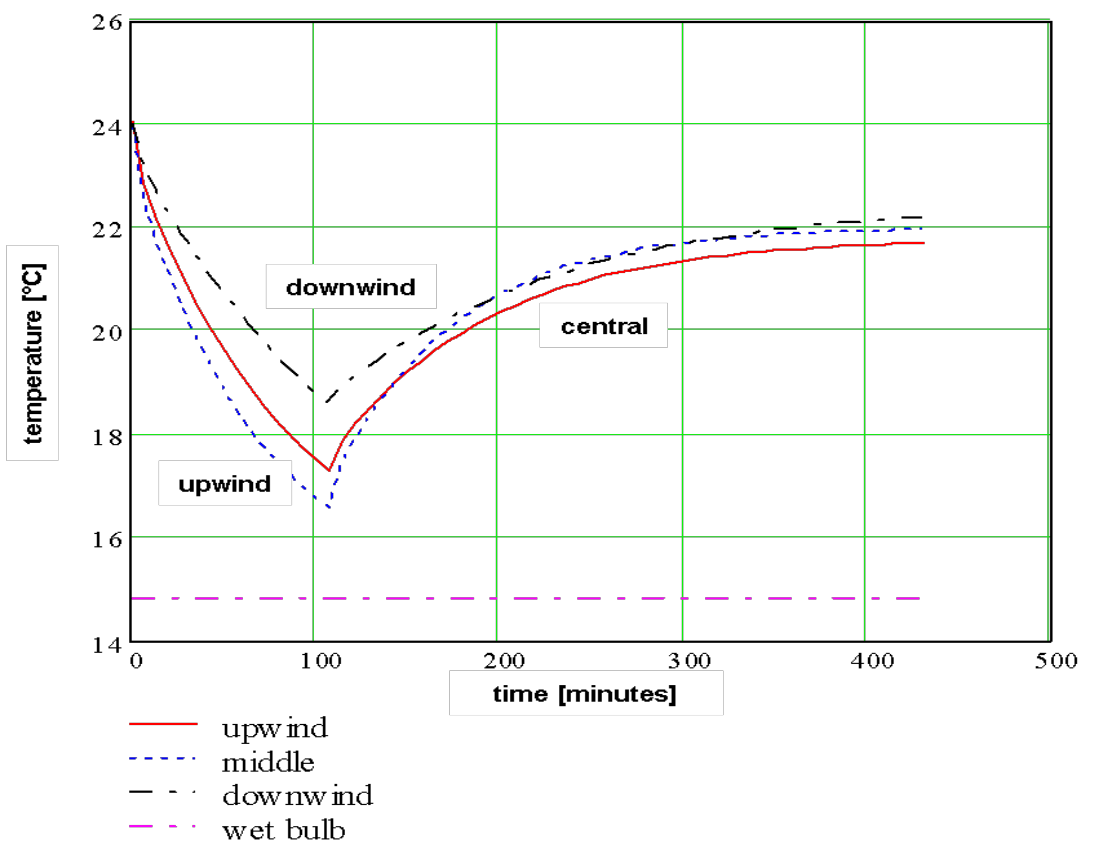

Fig. 9: Calculated proceed of temperature at three local points on the surface of a peach during drying off surface moisture

\section{Conclusion}

The primary effects on the moisture loss in a fruit or potato are the diffusion processes in the ambient air and in the produce material. The environmental conditions can easily be obtained under test. The analytical model gives a tool to approximate the boundary layer thickness for the mass transfer from the fruit surface of a transpiring produce to the ambient air. The air flow model using the method of CFD is used for the determination of air velocity distribution along the spherical fruit surface and across the boundary layer. From there, the calculation of the local mass transfer coefficient distribution along the surface and the total mass transfer coefficient becomes possible. The model in conjunction to surface temperature measurement, using thermal imaging, gives the possibility to predict heat and mass transfer for local differences of air flow around the surface of a fruit body. It is demonstrated from the model that the infrared thermographic camera is not measuring the wet bulb temperature form a drying surface but the real non-adiabatic evaporating process.

\section{REFERENCES}

[1] Linke, M. (1997a). Modelling of Heat Transfer in Individual and Packed Chicory Crops. Proc. of the COST $915-$ Copernicus CIPA-CT94-0120 Workshop on Food Quality modelling.

[2] Linke, M. (1997b). Modelling and Predicting the Postharvest Behaviour of Fresh Vegetables. Mathematical and Control Applications in Agriculture and Horticulture (Munack and Tantau, Eds.), Pergamon Press, Oxford, UK, 283288.

[3] Baehr H. D., Stephan, K. (1994). Heat and Mass Transfer, 2006. Springer-Verlag, Berlin Heidelberg.

[4] Hellebrand, H.J.; Beuche, H.; Linke, M.; Herold, B; Geyer, M. (2001): Chances and Shortcomings of Thermal Imaging in the Evaluation of Horticultural Products. International Conference "Physical Methods in Agriculture Approach to Precision and Quality", Prague 27-30 August 2001, Proceedings S. 112-117 (ISBN 80-213-0836-2)

[5] Fito, P. J.; Ortolá, M. D.; De Los Reyes, R.; Fito, P.; De Los Reyes, E., (2004): Control of citrus surface by image analysis of infrared thermography. Journal of Food Engineering, Vol. 61 (3), 2004, 287-290. 